\title{
Experiencias en micro y nano escalas para niños y jóvenes
}

\author{
Darwin D. Rodrícuez Pinto* \\ Alba G. Ávila Bernal**
}

\begin{abstract}
RESUMEN: Un nuevo laboratorio de nanotecnología donde niñas, niños y jóvenes aprenden sobre el micro y nano mundo abre sus puertas para motivar el aprendizaje de escalas, caracterización de materiales y técnicas de fabricación. Con la implementación de estrategias pedagógicas: un hacer práctico y un entender teórico, el uso de múltiples analogías comparativas con situaciones de su vida diaria y exploración de la naturaleza, en actividades interactivas se forma y capacita desde temprana edad a una masa semilla para el apoyo del avance científico en Colombia.

PALABRAS CLAVE: educación, nanotecnología, microscopios.
\end{abstract}

SUmmarY: A new nanotechnology lab where children and young people learn about the micro and nano world opens its doors to encourage learning of scales, materials characterization and fabrication techniques. The implementation of pedagogic strategies includes: practical activities and theoretical understanding, the use of multiple comparative analogies with situations in daily life and nature exploration, as well as interactive activities to learn and train a seed mass at an early age with the purpose of supporting scientific progress in Colombia.

KEY wORDS: education, nanotechnology, microscopes.

Desde febrero de 2010 el primer laboratorio de nanotecnología para niñas, niños y jóvenes de grado sexto a noveno, reconocidos en Colombia dentro del Servicio Nacional de Aprendizaje (SENA) como aprendices, bajo el proyecto llamado Tecno Academia, abrió sus puertas en la zona industrial de Cazucá, localidad del sur de Bogotá. Con una inversión y donaciones del orden de $\$ 25,000,000,000$ pesos, en este laboratorio se explora la materia a una escala de $10^{-9} \mathrm{~m}$ educando alrededor de 1,000 niñas y niños colombianos, fuera del contexto tradicional de educación centrado en universidades y centros de investigación.

El entrenamiento de 1,000 niños y jóvenes es significativo si se considera que al 2011 sólo instituciones de educación superior públicas han tenido una convocatoria de similar magnitud en cátedras de formación Integral como es el caso de la Cátedra José Celestino Mutis "Nanotecnología, el tamaño sí importa" que actualmente ofrece la Universidad Nacional de Colombia (una de las más reconocidas a nivel nacional) y que incluye virtualmente a estudiantes de otras sedes nacionales como son Medellín y Manizales (Mutis, 2011).

Los aprendices que visitan el laboratorio en dos sesiones semanales en contra jornada escolar, no requieren conocimientos previos, sin embargo, se les aplica un examen de clasificación basado en los modelos de pregunta de las pruebas Saber 2009.

\footnotetext{
* Estudiante de maestría, Universidad de los Andes. Líder en Nanotecnología Tecno Parque Nodo Cazucá. <dd. rodriguez89@uniandes.edu.co>.

** Profesora Asociada. Departamento de Ingeniería Eléctrica y Electrónica. Universidad de Los Andes. <a-avila@ uniandes.edu.co>.
} 
En estas sesiones se hace uso de los recursos e infraestructura que comprenden: un microscopio de barrido de fuerza atómica, un microscopio de barrido electrónico, microscopio de fluorescencia y un nano-litógrafo. Estos recursos son utilizados para la enseñanza de conceptos que se consideran vitales en un proceso de formación básica en micro y nanotecnología para estos aprendices: escalas de trabajo, caracterización topográfica, propiedades de materiales y fabricación. Los conceptos son transmitidos a los aprendices a través de actividades que enfatizan: un hacer práctico y un entender teórico (HPET), el uso de múltiples analogías comparativas con situaciones de su vida diaria (MAV) y exploración de la naturaleza (biosistemas naturales (BS)). Estas estrategias tienen como objetivo exponer al aprendiz a conceptos desde su quehacer diario, explorando lo que el aprendiz "ve" y le es tangible a su alrededor para entender las dimensiones de trabajo a escalas nanométricas; la importancia de herramientas como microscopios para la "observación", manipulación y entendimiento de propiedades que naturalmente pueden encontrar en su entorno (hidrofobicidad). La motivación se evidencia en los aprendices por el incremento en el número de preguntas que surgen sobre si otros materiales pueden mostrar estas propiedad de hidrofobicidad, la relación directa que hacen con aplicaciones de limpieza y protección a la actual crisis invernal en ropa y accesorios como gafas, y su constante interés en extender las horas de trabajo en la tecno academia. Identificar escalas, propiedades de materiales, métodos de fabricación a nanoescala dan a los estudiantes habilidades básicas que sugerimos como base para desarrollar competencias en futuros nanotecnólogos a temprana edad y que no directamente en nanotecnología, ya son de forma similar aplicadas en Estándares Básicos de Competencias en Ciencias Naturales y Tecnología establecidas por el Ministerio de Educación (Educacion, 2004).

A continuación se hace una descripción de las experiencias interactivas que se desarrollan con los aprendices para transferir los conceptos con las herramientas disponibles enfocados en las estrategias pedagógicas HPET, MAV y BS.

\section{EsCALA}

Una manera de dar a entender y de comprender a qué escala se está trabajando (graduación empleada en diversos instrumentos para medir una magnitud (Lengua, 2011), especialmente cuando se trabaja en algo tan pequeño $\left(10^{-9}\right.$ metros $)$, es hacerlo a través de las perspectivas: convencional, ilustrativa y práctica.

1. Convencional: con el uso de una regla que exprese sus métricas en nanometros, como aparece en la figura 1, se introduce al aprendiz a despertar una intuición de lo pequeño a lo grande. ¿Por qué la regla?, porque es el elemento que todos los aprendices dominan en su rutina escolar. Aquí se enfatiza en el hacer práctico y el entender teórico solicitando a los aprendices la medición de objetos comunes como es el caso de un perro y una pulga en nanómetros.

2. Ilustrativa: a partir de videos interactivos se expone al aprendiz a una perspectiva de escala en diferentes entornos desde la escala macro hasta la fotométrica, motivando su curiosidad por ver componentes de la materia en elementos de la vida diaria (carros, insectos y cuerpo humano) y enfatizando así la estrategia pedagógica MAV. (Reisen, 2011) (Europea, 2005). 
FIGURA 1. Regla con métricas en nanómetros y sus mediciones en seres comunes como el perro y una pulga
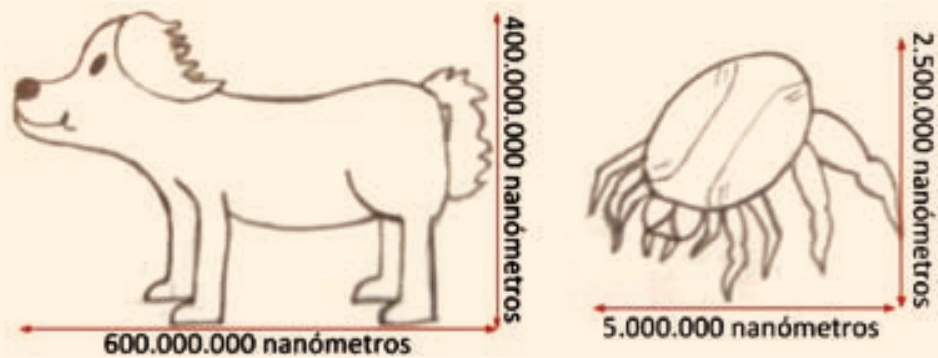

Fuente: Elaboración propia.

3. Práctica: a micro escalas, la práctica se concentra en visualizar insectos con el apoyo de microscopía de barrido electrónico (SEM), ver imagen 1. A través del ejercicio de usar un SEM, el estudiante va de la práctica al entender del concepto de escala micro determinando el tamaño de algunos de los detalles en el cuerpo del insecto. Detalles que no son observables con sus ojos y que hacen también conciencia de la necesidad y relevancia de microscopios de barrido como instrumentos de apoyo para visualización a diferentes escalas.

IMAGEN 1. Fotografía tomada con microscopio de barrido electrónico a un insecto

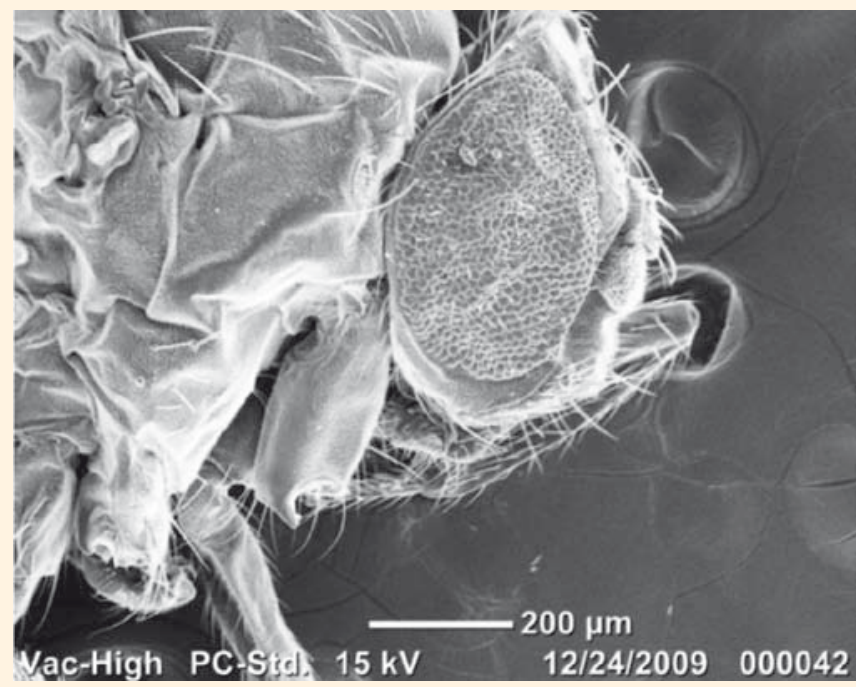

Al final de esta sesión los aprendices de Tecno Academia comprenden la escala de trabajo (de $10^{-6}$ metros a $10^{-9}$ metros), miden diversos elementos de su entorno (hacer práctico) y los relacionan con prefijos numéricos (el entender teórico) y términos como micro y nanotecnología y micro y nanoescalas. 


\section{Caracterización}

En las prácticas de caracterización es primordial que los aprendices conozcan los instrumentos que hacen posible la manipulación, visualización, modificación y caracterización de la materia a nanoescalas. Aquí son introducidos los microscopios de barrido.

Usando el microscopio de fuerza atómica (AFM, por sus siglas en inglés) para la medición de muestras con patrones nanométricos, se introduce a los aprendices a los fundamentos del AFM, su operación, sus limitaciones y requerimientos (ver imagen 2). El objetivo aquí es usar muestras de fácil accesibilidad como es un CD. En la imagen 3: "Topografía de un CD de datos tomada con el AFM". Se muestra la imagen sobre la cual los aprendices definen formas, separaciones y dimensiones de patrones entre pistas. Esta muestra, además, permite ilustrar a los aprendices cómo la información digital es almacenada como documentos, videos, música, entre otros datos, y se les explica cómo se representa esta información en bits, que es la unidad más pequeña de información.

IMAGEN 2. Niñas, niños y Jóvenes usando y aprendiendo con el AFM

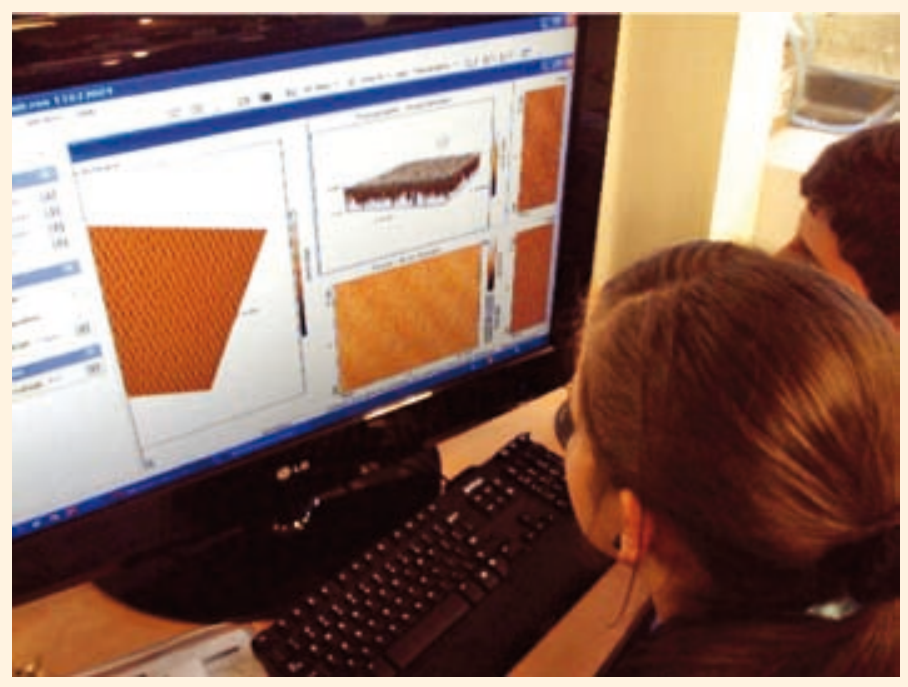

En esta actividad se evalúa la comprensión de las nanoescalas introducidas en las prácticas iniciales y a partir de aquí se avanza en el uso del microscopio para estudiar propiedades a nanoescala como es la hidrofobicidad (capacidad de un material de repeler el agua y no permitir la adherencia en éste.)

\section{Propiedades}

El manejo del AFM permite a los aprendices investigar el porqué de algunas propiedades de la materia, específicamente hidrofobicidad (ver imagen 4). Con el análisis de muestras encontradas en el mismo TecnoParque como polillas e insectos se analizan las estructuras sobre las muestras y el rechazo del agua sobre las mismas. 
IMAGEN 3. Topografía de un CD de datos tomada con el AFM

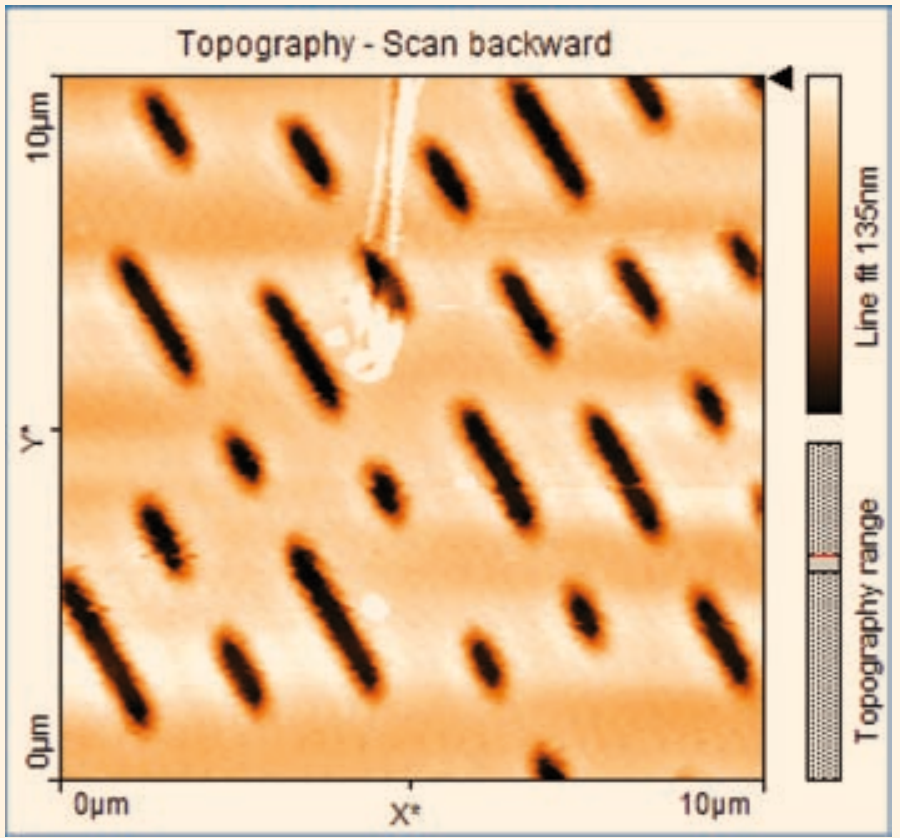

IMAGEN 4. Niñas, niños y jóvenes evaluando hidrofobicidad en diversos materiales

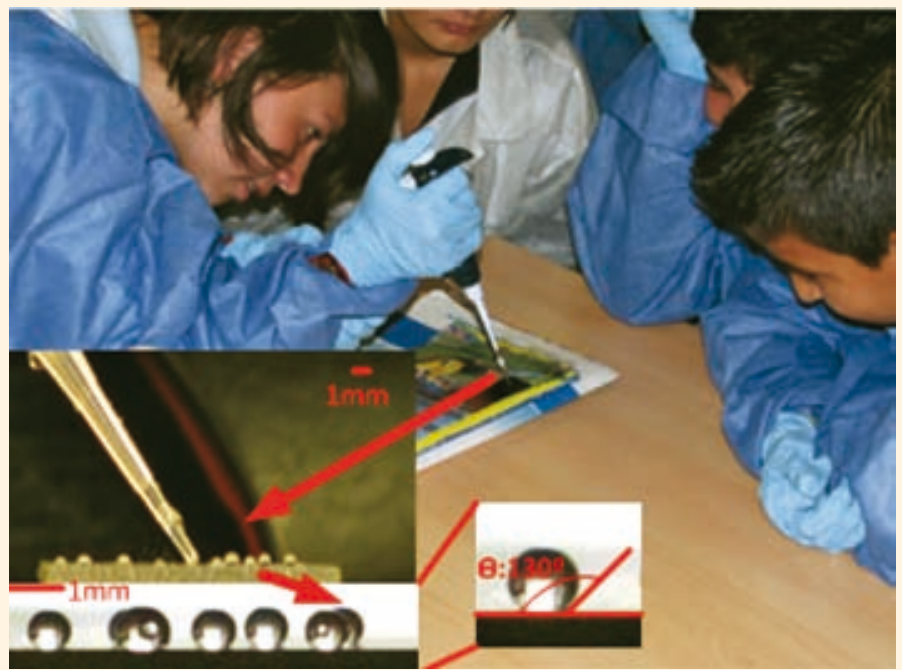


En esta actividad, los aprendices se familiarizan con mediciones de ángulos de contacto de gotas de agua sobre las muestras, ángulos mayores de $90^{\circ}$ representa hidrofobicidad y menores a $90^{\circ}$ hidrofilia (aceptación de agua de un material).

En la imagen 5 se muestra la topografía del ala de una polilla, en la cual se presenta hidrofobicidad gracias a la estructura en malla que guarda distancias equidistantes entre 25 um y 30 um, seguramente estas estructuras tienen relación con las características hidrofóbicas de estos biomateriales. Esta actividad está ligada a estrategias pedagógicas HPET y BS.

IMAGEN 5. Topografía del ala de la polilla en su parte interna, esta estructura muestra súper hidrofobicidad.

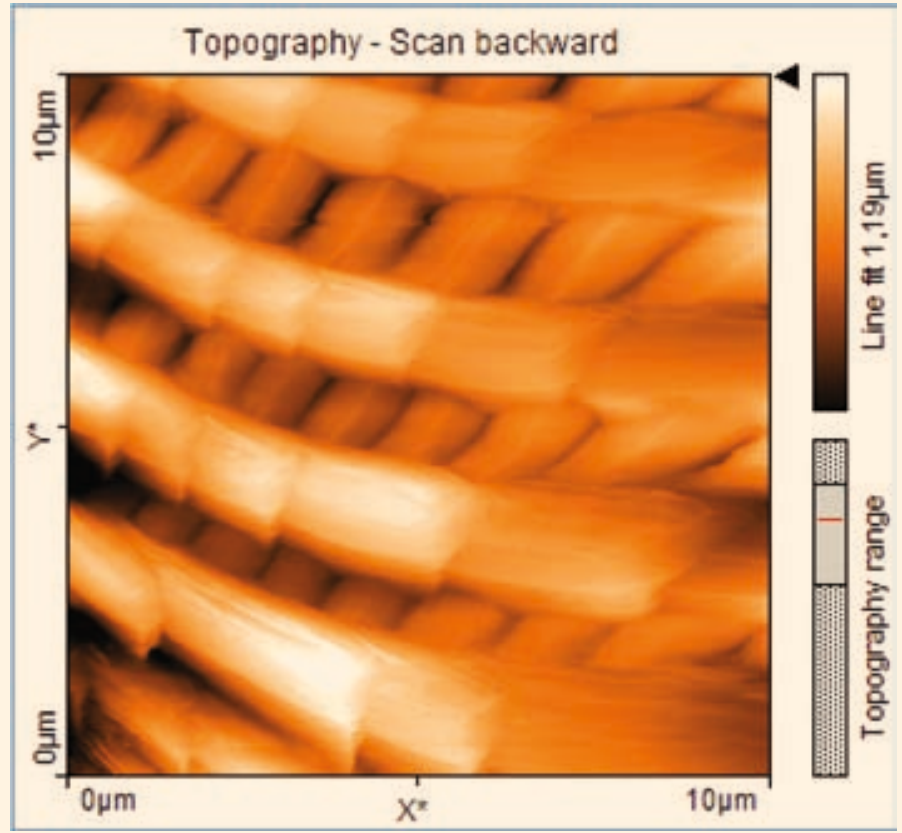

\section{FABRICACIÓN}

Por medio del uso de nano litografía con el equipo NLP2000 (NanoInk, 2010), los aprendices ubican una plataforma que les permite mover arreglos de puntas en los ejes X y Y como se muestra en la imagen 6. Con esta plataforma se fabrican arreglos de puntos y líneas a microescalas que no son visibles a simple vista y así el aprendiz reconoce la necesidad del uso de la microscopía (ver imagen 6).

También, como practica de fabricación se realiza litografía sobre películas delgadas como se muestra en la imagen 7 , lo cual ayuda a entender los diferentes métodos de fabricación y manipulación a estas escalas, usando analogías comparativas tal como si la mano que escribe es el NLP2000, el lápiz es el arreglo de puntas, y el papel donde escribimos es la película delgada donde se realizan los rayones, así se transfiere este concepto de forma apropiada y creativa usando estrategias como HPET. 
IMAGEN 6. Arreglo de puntas para litografía y deposición usando el Nano Litógrafo NLP2000

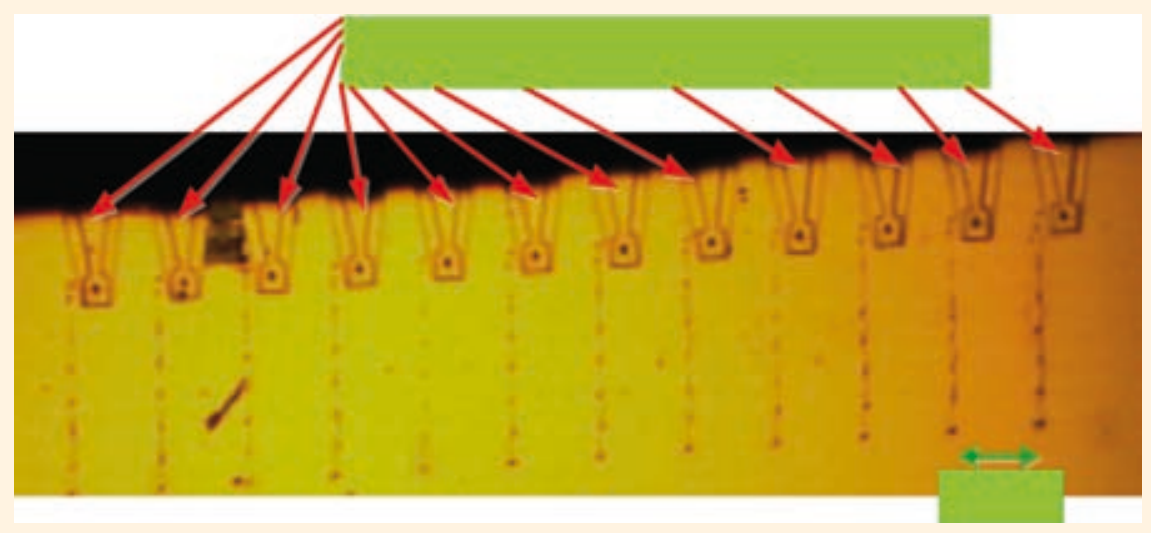

IMAGEN 7. Trazado y desbastado de zona con película delgada de aluminio. Fotografía tomada con microscopio de fluorescencia

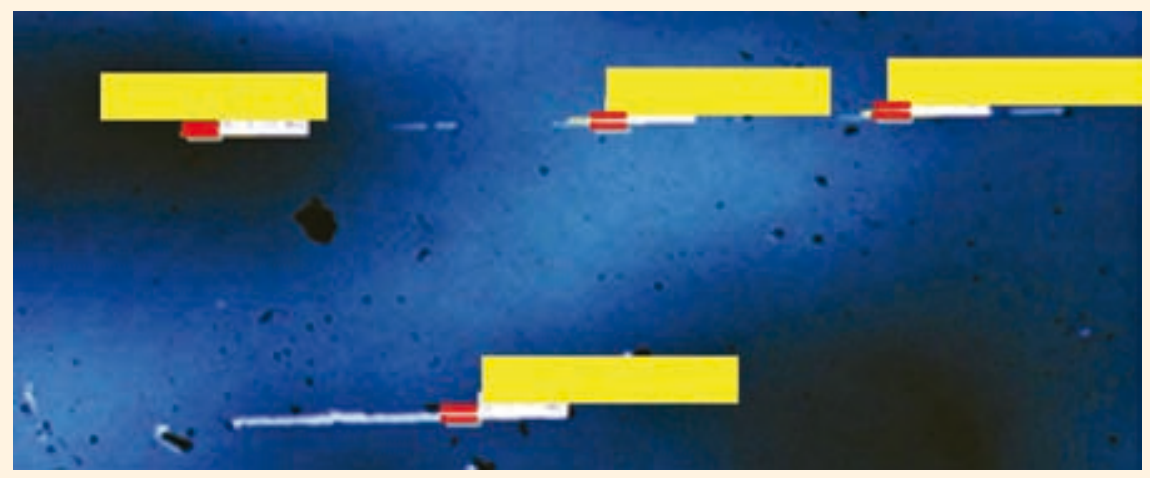

Con cada una de las actividades antes mencionadas: escalas, caracterización, propiedades y fabricación motivan y educan a los aprendices con habilidades y competencias en nanotecnología. El seguimiento realizado al final de las actividades demuestra que con el enfoque descrito en este artículo (HPET, MAV y BS), el 75\% de aprendices entiende el uso de prefijos del sistema métrico relacionados con dimensiones (nanómetro, micrometro) y escalas, además de, construir sobre estos prefijos otros términos como: nanoescalas, nanochips, nanorrobots, nanociencia y nanotecnología. En términos de caracterización el 86\% de los aprendices reconocen e identifican el lenguaje técnico o siglas técnicas asociadas a los microscopios: óptico, de fuerza atómica y de barrido electrónico. Conceptualmente, ellos también relacionan el principio físico que permite el funcionamiento de cada microscopio: luz, electrones y cantiléver interactuando con la muestra bajo estudio. A nivel de propiedades, el 36\% de los aprendices comprenden el concepto de hidrofóbicidad, lo relacionan con biomateriales estudiados dentro y fuera la práctica desarrollada en la Tecno Academia; sin embargo, sólo un pequeño porcentaje de 12\% reconoce cómo puede verificar si un 
material es o no hidrofóbico. Nuevas prácticas y proyectos para enfatizar métodos de verificación están en proceso de diseño.

\section{Conclusiones}

El laboratorio de nanotecnología del Tecno Parque Central del SENA es un espacio complementario de formación y capacitación temprana en conceptos básicos en micro y nanotecnología fuera de los contextos convencionales de formación académica. Estos conceptos son transferidos a los aprendices de una manera dinámica con las actividades antes mencionadas, guiando a los aprendices en la construcción de sus conceptos y destacando el uso de estrategias pedagógicas como hacer práctico y aprender teórico, el uso de múltiples analogías comparativas con situaciones de su vida diaria y exploración de la naturaleza que luego ellos puedan utilizar en el desarrollo de proyectos y en su continua formación a titulaciones como técnico o tecnólogo en nanotecnología. Los equipos, las experiencias y los resultados aquí descritos en escalas, caracterización y fabricación hacen de este laboratorio el único en Colombia con infraestructura y capacidad de formación temprana. Actualmente, experiencias similares sólo están disponibles en centros de investigación y en universidades a nivel nacional como la Universidad Nacional y la Universidad de los Andes, quienes son los que cuentan con laboratorios e infraestructura similar a Tecno Parque. El reto para el SENA y el gobierno es, entonces, mantener y continuar la capacitación de nuestras niñas, niños y jóvenes micro y nanotecnólogos.

Finalmente, este tipo de proyectos educativos van de la mano con los planes de desarrollo del gobierno nacional como el programa "De cero a Siempre", cuyo objetivo principal es apoyar la primera infancia en su proceso educativo (Nacional, 2011). Es importante dejar en claro que este esfuerzo tiene un efecto incalculable en la formación por competencias en nanotecnología de nuestras niñas, niños y jóvenes que se espera aporten en su proyecto de vida y que el gobierno debe responsabilizarse por generar programas que den continuidad a niveles más avanzados de educación.

\section{Agradecimientos}

SENA (Servicio Nacional de Aprendizaje) TecnoParque y Tecno Academia Nodo Cazucá, "Por un proyecto que siembra futuro".

\section{Trabajos Citados}

1. Gobierno Nacional, (2011), "Programa de Cero Siempre: <http://www.deceroasiempre.gov.co>.

2. Mutis, C. J. (2011). “Cátedra de Nanotecnología (El tamaño sí importa). Universidad Nacional De Colombia": <http://www.catedras-bogota.unal.edu.co/mutis/ 2011-I/>.

3. Ministerio de Educación. (2004). "Estándares Básicos de Competencias en Ciencias Naturales y Ciencias Sociales".

4. NanoInk. (2010). "Plataforma de Nanolitografía": <http://www.nanoink.net/>

5. Real Academia de la Lengua. (2011). "Definiciones". s.l. : <http://drae2.es/escala>.

6. Reisen. (2011). "Nano Reisen": <http://www.nanoreisen.de/>.

7. Unión Europea. (2005). “Un Viaje Por el Nano Mudo” [película]. 